OPEN

SUBJECT AREAS:

NANOCAVITIES

NANOPHOTONICS AND

PLASMONICS

SURFACE PATTERNING

OPTICAL PHYSICS

Received

20 September 2012

Accepted

14 November 2012

Published

6 December 2012

Correspondence and requests for materials should be addressed to A.P. (apolyakov@|bl. gov)

\section{Plasmon resonance tuning in metallic nanocavities}

\author{
A. Polyakov, K. F. Thompson, S. D. Dhuey, D. L. Olynick, S. Cabrini, P. J. Schuck \& H. A. Padmore
}

Lawrence Berkeley National Laboratory, 1 Cyclotron Road, Berkeley, CA, USA.

Nanocavities fabricated in a metallic surface have important and technologically useful properties of complete light absorption and strong field enhancement. Here, we demonstrate how a nanometerthick alumina deposition inside such a cavity can be used to gain an exquisite control over the resonance wavelength. This process allows achieving a precise control over the spectral response and is completely reversible allowing many tuning attempts to be made on a single structure until the optimum performance is achieved.

ight trapping on metal surfaces has a long history ${ }^{1-6}$ and has been demonstrated in a wide variety of structures for applications ranging from light filtering ${ }^{7,8}$ to biosensing ${ }^{9}$ to harmonic generation ${ }^{10-13}$. Structures such as lamellar gratings, split-ring resonators, plasmonic coaxial guides and bowties, among others, have been investigated in great detail both theoretically ${ }^{6,14-18}$ and experimentally ${ }^{19-24}$. A special form of these light trapping nanostructures that can achieve strong broadband absorption and field enhancement is the subwavelength groove nanocavity (NC). However, structures composed of NCs resonant in the visible spectrum have a characteristic dimension ${ }^{6}$ on the order of $10 \mathrm{~nm}$, which makes realizing the device with an exact resonance wavelength a fabrication challenge. In this work, we present a new method for tuning the plasmon absorption resonance wavelength with a nanometer precision anywhere from the visible (VIS) to the near infrared (NIR) in postfabrication by deposition of a few nanometers of aluminum oxide inside the grooves via atomic layer deposition (ALD). Furthermore, in order to achieve a high field enhancement within the NCs in the NIR spectrum, the NC must have a higher aspect ratio ${ }^{21}$ with the width of around $15 \mathrm{~nm}$. In such a case, the ALD-based plasmon resonance tuning becomes a powerful technique for preparing a device that would not otherwise be possible by direct fabrication. Additionally, the ALD coating provides robust protection against surface contamination and can be used to prevent oxidation in such metals as silver and copper.

Light trapping in the visible spectrum via a subwavelength array of rectangular NCs has been proposed theoretically by Le Perchec et al. ${ }^{6}$ and demonstrated experimentally in our recent work ${ }^{25}$. The schematic for such a grating is shown in figure 1(a): on resonance, light polarized perpendicular to the orientation of the NCs (denoted p-polarization) excites a standing wave surface plasmon polariton (SPP) mode within the NC, producing the electric field pattern as illustrated in figure 1(b). This SPP mode has its strongest electric field at the mouth of the groove and strongest magnetic field at the bottom. The field enhancement achieved at the mouth of the groove is advantageous for nonlinear optical applications such as harmonic generation and multiphoton photoemission ${ }^{25}$. In this way, the NCs serve both as an SPP coupling mechanism, as well as resonant cavities with strong field enhancement ${ }^{26}$ for absorption at the resonant wavelength $\lambda_{\text {res }}$.

Figure 1(c) shows the reflectivity spectrum of such a grating, measured at normal incidence; the black solid curve shows the corresponding numerical FDTD calculation. The grating was designed by numerically optimizing the period $p$, width $w$ and depth $h$ of the NCs to produce a resonance in the VIS-NIR region. In this case, for a grating made on a gold surface: $\lambda_{\text {res }}=720 \mathrm{~nm}, w=13 \mathrm{~nm}, h=42 \mathrm{~nm}, p=600 \mathrm{~nm}$. The reflectivity was measured in reference to the s-polarized light, which does not excite the SPP modes inside the NCs and, therefore, has the response of the bulk material. Two reflectivity dips are observed in the reflectivity spectrum shown in figure 1(c). The broader dip, at $720 \mathrm{~nm}$, is due to the resonant SPP modes excited within the NCs, which experimentally yields a 16 times absorption increase compared to the plain gold surface. Theoretically, the reflectivity can be reduced to below $0.01 \%$ for NCs with perfectly square edges. In contrast, the classical grating-coupled SPPs are excited on the top surface ${ }^{27}$, and propagate in between the NCs. The narrow reflectivity dip at $600 \mathrm{~nm}$ corresponds to this classical grating-coupled SPP mode. For photovoltaic and ultrafast applications it is desirable to produce large spectral bandwidth light trapping substrates ${ }^{28}$, while for other applications, such as sensors $^{29}$, narrower bandwidth is preferred. Both types of response can be achieved by adjusting the $p / \lambda_{\text {res }}$ ratio $^{30}$. 
(a) P-polarization

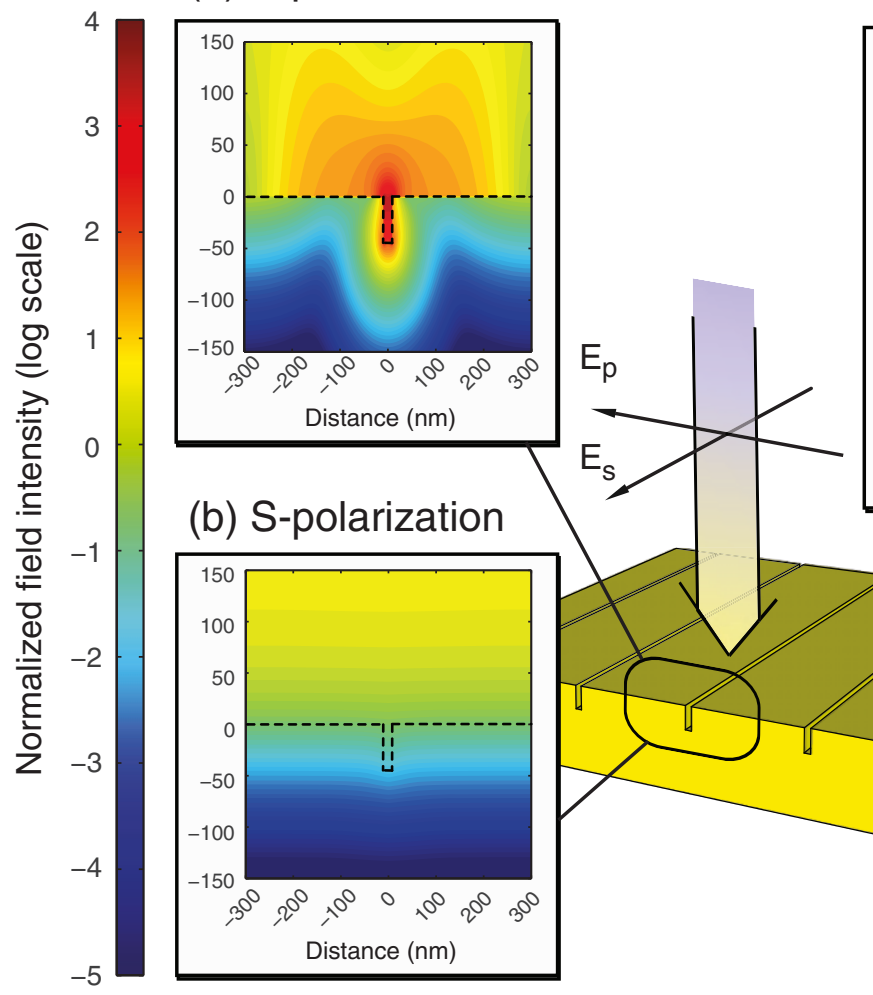

(c) Optical response

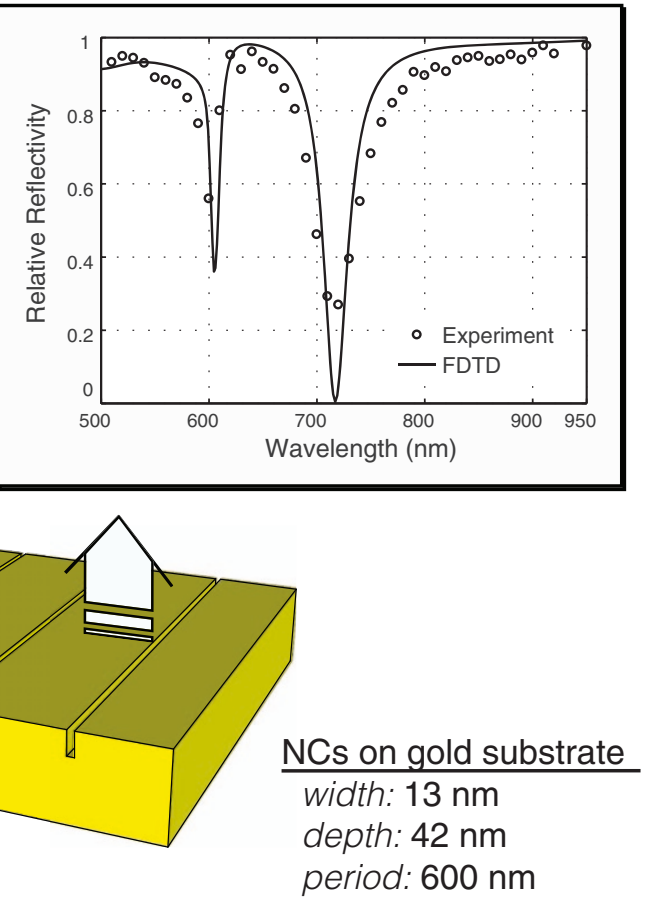

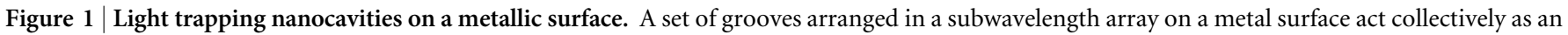

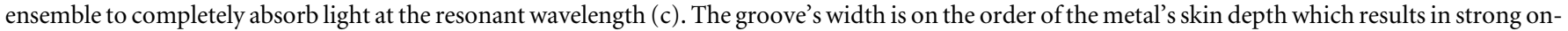

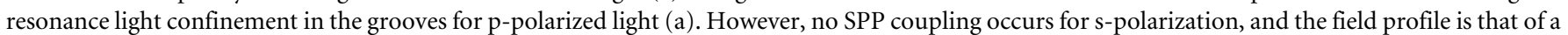
bulk material (b).

It has been previously shown that for $150 \mathrm{~nm}$ in diameter hole structures, a conformal dielectric coating can be used to effectively tune the transmission resonance as well as to enhance the chemical stability of the plasmonic structure ${ }^{38}$. Additionally, ALD coating is now emerging as a powerful tool to provide functionalized surfaces of increased sensitivity in bio-sensor applications ${ }^{39,40}$. In this work for the first time, we show that ALD can be used at a much smaller scale to tune $10 \mathrm{~nm}$ sized cavity structures in a completely reversible manner. In the case of a deep subwavelength grating, a small amount-less than a nanometer thick layer-of a dielectric on the cavity surface shifts the resonance position by more than $10 \mathrm{~nm}$. The dielectric layer on the metal surface changes the boundary condition for the SPP dispersion ${ }^{41}$, shifting the resonance wavelength for the SPP standing wave excited in the NC. The dispersion relations for plasmons propagating in a Metal Insulator Metal (MIM) cavity are well known ${ }^{31}$ and show that not only does the wave vector of the plasmon travelling in a cavity depend on the metal dielectric function; it also depends on the dielectric function of the insulator that separates the two sides of the cavity. Normally this cavity is empty, so filling it with a dielectric material naturally leads to a mechanism for tuning the plasmon wave vector and hence the position of the absorption maximum. The wavelength position of the absorption maximum can be calculated directly from the normal dispersion relations of MIM cavities. This effect was successfully used in the fabrication process to test for the presence of surface contaminants on the gold grating discussed above.

\section{Results}

The sensitivity of the plasmonic grating to the presence of a dielectric coating was effectively used to tune the grating absorption resonance wavelength in post-fabrication. A well-controlled thickness of the dielectric coating was conformally deposited onto the structure by an ALD growth process, yielding a high-quality conformal coating $^{32-34}$.

Due to the narrow opening of the NCs the alumina layer growth was non uniform inside the NCs with the middle of the NC filled with a less dense material then at the edges of the NC. The transmission electron microscopy (TEM) image showing the cross section view of the completely filled NC is shown in figure 2(a). While the topsurface alumina coating has uniform density and appears of constant shade, the alumina filling of the NC varies greatly in shade corresponding to the density variation of the alumina filling: as the dimensions of the cavity approach values less than $10 \mathrm{~nm}$ in width, the alumina growth is no longer uniform and instead forms a less dense material.

The optical properties of the alumina filling were modeled by approximating the non-uniformity of the alumina growth inside the NCs as an exponential decay in the index of refraction of the alumina filling corresponding to the average intensity variation inside the NC as indicated in figure 2(a) and plotted in figure 2(b). The refractive index is modeled to vary from the nominal value of $n_{o x}$ $=1.6$ at the $\mathrm{NC}$ edge to the reduced refractive index at the center of the NC, $n_{c}$, which is treated as a fit parameter. The mathematical model for the alumina index of refraction is then given by:

$$
n(d)=\left(n_{o x}-n_{c}\right) \exp [-d / t]+n_{c},
$$

where $d$ is the distance away from the edge of the groove, and $t$ is the exponential decay length; figure 2(c) shows the plot of the modeled index of refraction inside the NCs. Two gold gratings of closely matched dimensions were prepared to measure the dielectric-based tuning: sample A with $w=13 \mathrm{~nm}, h=42 \mathrm{~nm}, p=600 \mathrm{~nm}$, and sample B with $w=15 \mathrm{~nm}, h=42 \mathrm{~nm}, p=600 \mathrm{~nm}$. Grating A was 

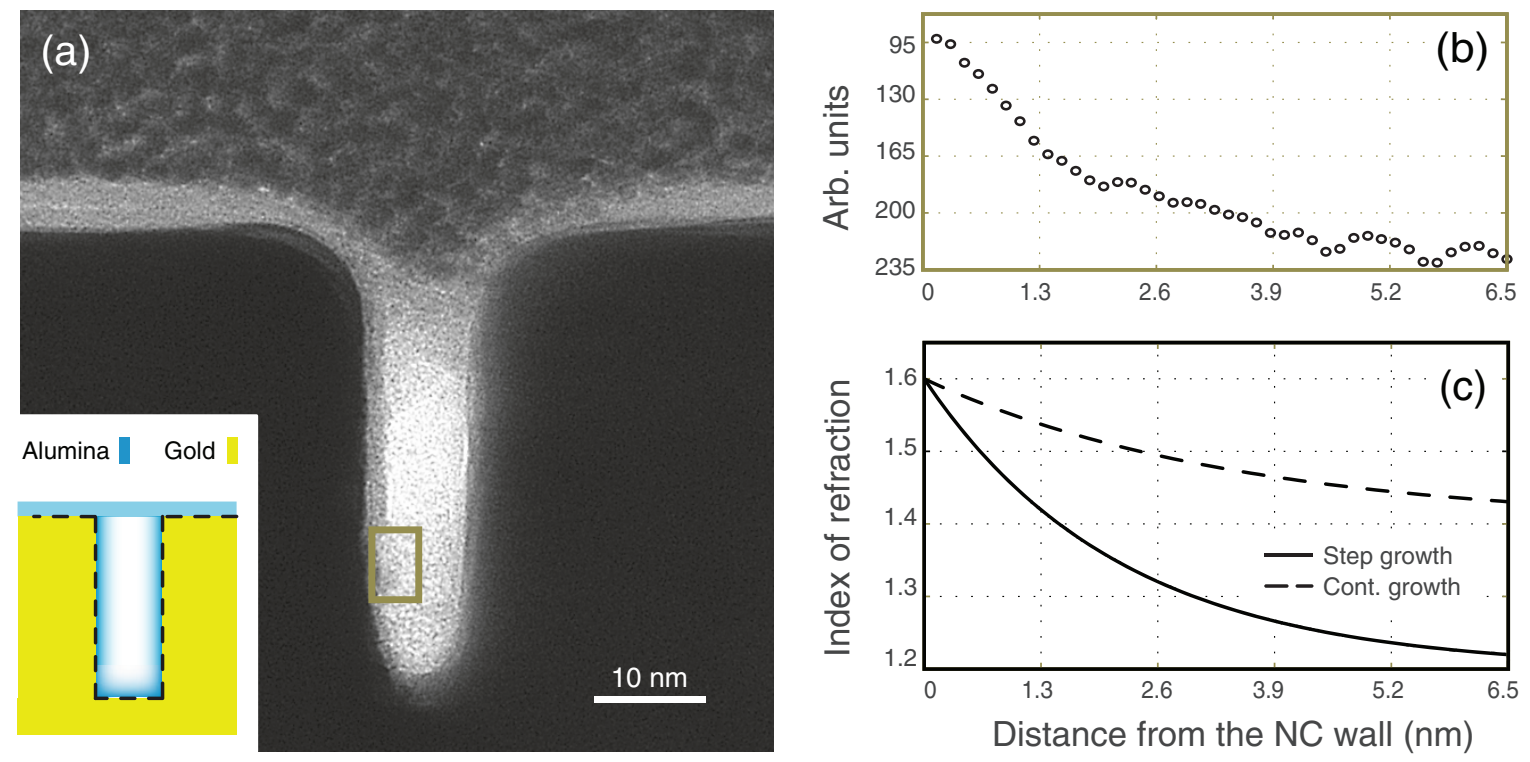

Figure $2 \mid$ Alumina composition variation within the nanocavity. The TEM image of the filled NC shows a variation in the alumina density from the NC wall to the middle of the groove. This variation was used as a basis for the FDTD model: plotting the average pixel intensity-integrated over a region indicated by a rectangle in (a)-yields an exponential decay curve (b). The alumina index of refraction is therefore modeled as an exponential decay function from the nominal alumina refractive index of 1.6 to a lower value at the center of the NC (c). The decrease in the value of the refractive index is attributed to the alumina density decrease away from the cavity walls.

designed for resonant absorption at around $705 \mathrm{~nm}$ wavelength with the reflectivity spectrum shown in figure 3(a). The structure was then coated with 20 cycles of alumina $\left(\mathrm{Al}_{2} \mathrm{O}_{3}\right)$ ALD growth resulting in the resonance shift past $815 \mathrm{~nm}$. The alumina was then removed by an hydrofluoric acid (HF) sample cleaning, which returned the grating to its original state-see figure 3(a). The same process was repeated for 50 cycles of ALD growth producing a resonance shift of $160 \mathrm{~nm}$ at $865 \mathrm{~nm}$ starting from the original resonance at $705 \mathrm{~nm}$.
Using the process described above the resonance position was measured as a function of the alumina thickness as shown in figure 3(b). For sample A the alumina was first grown via 2, 4, or 8 ALD cycles in a deposition; then the sample was taken to air to measure the reflectivity spectrum. A new layer of alumina was then incrementally grown. Blue circles in figure 3(a) correspond to each measured resonance position; the solid line is the FDTD model fit based on the dielectric constant as described above (the steps in the (a) Resonance shift

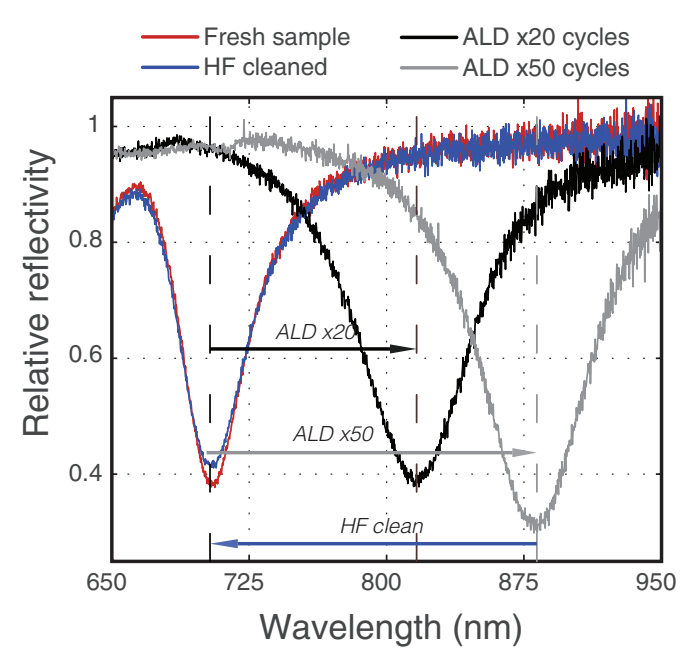

(b) Resonance tuning

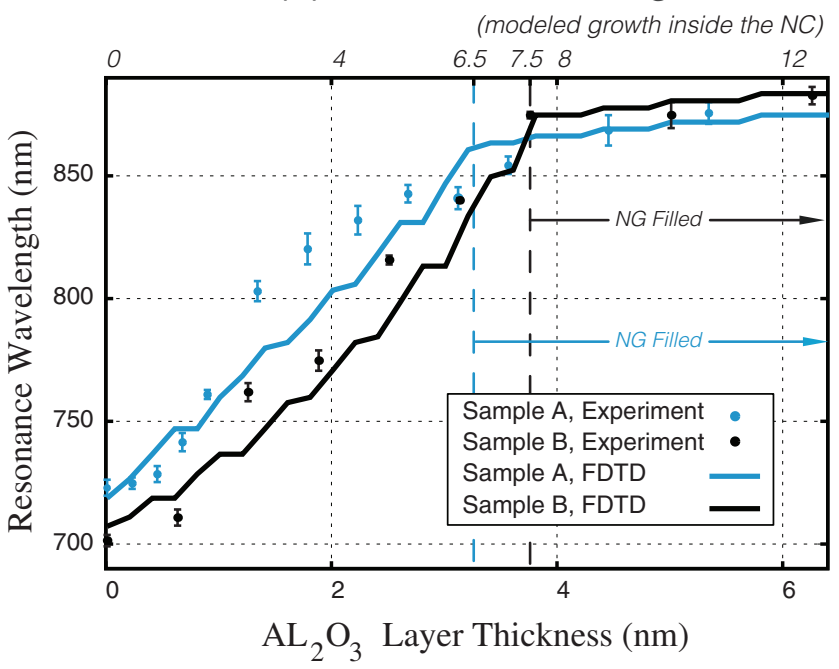

Figure 3 Plasmon resonance tuning. Depositing a layer of alumina inside the NCs strongly affects the resonance wavelength. The original response, however, can be fully recovered by etching out the alumina; (a) shows four plots: the original reflectivity spectrum, the spectrum after 20 cycles of alumina ALD compared to 50 cycles, and a plot of the recovered original response after HF cleaning. This effect allows tuning of the plasmon resonance in post fabrication (b). Two gold gratings, samples A and B, were prepared: for sample A, the alumina growth was incremental, with the sample exposed to air in between each successive deposition; for sample B, the alumina was grown in one deposition from $0 \mathrm{~nm}$ to the target thickness, then alumina completely removed returning the resonance to the original wavelength. And the entire process repeated. Both samples are tuned from the resonance position up to $\approx 860 \mathrm{~nm}$. This effect saturates once the NC is completely filled-this critical alumina thickness is marked by the vertical dashed lines in (b). 
theory curve are due to the finite size of the mesh used). When the sample was taken out to air, a reacted layer would form that resulted in the overall alumina growth to be less dense yielding $n_{c}=1.2$ and $t$ $=2.5 \mathrm{~nm}$. The growth rate on the top surface was measured to be 1.1 $\AA$ /cycle based on the TEM picture shown in figure 2(a). The growth inside the NC is modeled to be twice that amount $(2.2 \AA /$ cycle $)$ as discussed above in good agreement with the TEM cross section showing the light region of the alumina in the center of the NC.

For sample B, the alumina was grown to the target thickness all in one deposition starting from a completely cleaned surface each time. After measuring the resonance position at the target alumina thickness, the sample was cleaned using a combination of the HF, acetone, and isopropyl alcohol (IPA), and the resonance position of the cleaned sample was consistently confirmed to be $\lambda_{0}^{\text {res }}=705 \pm 2 \mathrm{~nm}$ before growing the new target thickness of the alumina layer (no appreciable change in the surface quality of the nanopattern was evident in the SEM images). This way, a total target thickness of alumina was deposited onto the clean structure without any contamination. This process was repeated nine times and the result is plotted in figure 3(a) (black squares) with the corresponding FDTD model. Alumina grown this way yielded a more dense coating with the FDTD model fit parameters of $n_{c}=1.4$ and $t=4 \mathrm{~nm}$. Unlike sample A, sample B was not exposed to air in between the successive layers, therefore the alumina growth rate is slightly higher, $1.25 \AA$ /cycle on the top surface, or $2.5 \AA$ /cycle inside the NC, which is consistent with growth rates reported for alumina ALD at room temperate under similar conditions ${ }^{34-36}$.

Both samples exhibit the same tuning behavior, in good agreement with the FDTD: in the region where the NC is not completely filled, sub nanometer size increase in the thickness of the dielectric layer partially filling the NC produces a large shift in the resonance position. In this regime, the resonance can be tuned anywhere up to the NIR region. Once the NC is filled, the tuning saturates. The thickness of the alumina layer at this point is half the NC width and this critical thickness is marked with vertical dashed lines in figure 3(a). Any continued growth is equivalent to a small decrease in the periodicity of the subwavelength grating yielding only a minor further shift in the resonance wavelength.

\section{Discussion}

ALD deposition of a conformal dielectric coating inside the metallic NCs allows a wide spectral bandwidth plasmon resonance tuning in a completely reversible manner. This process can be repeated many times-reverting to the original grating response by HF cleaning-in order to achieve the optimal response. This flexibility offered by the dielectricbased tuning is also useful beyond post-fabrication resonance adjustment. Preparing a plasmonic grating resonant in the NIR with large field enhancement requires extreme aspect ratio, which is a major fabrication challenge $e^{37}$. However, NIR resonance can be achieved by designing a grating for resonance towards the red part of the visible spectrum and later converting it into the NIR grating by controllable growth of a thin dielectric coating. This technique is completely reversible: the alumina is readily removed from the gold grating by an HF etch completely recovering the initial optical response. This effect also opens the possibility of using such gratings as sensors for probing sub-nanometer thick layers of a dielectric.

In summary, we have presented a design and post-fabrication tuning of a subwavelength plasmonic metallic grating for complete control over the resonance wavelength, the absorption strength, as well as spectral and angular bandwidths. Exquisite control over the resonance position was demonstrated in this study of gold gratings that were tuned in a completely reversible manner from a wavelength of $720 \mathrm{~nm}$ up to $860 \mathrm{~nm}$ by ALD growth of a few nanometer thick layer of alumina. This coating also serves as a protective layer for the metal structure, allowing fabrication of highly controlled resonant light trapping metal surface with resistance to environmental contaminants. Both types exhibit optical characteristics in good agreement with the FDTD model presented. Combining the design of the grating with the tunability offered by the dielectric coating allows production of a robust, completely absorbing substrate with a tunable resonance wavelength.

\section{Methods}

The plasmonic grating fabrication is based on the evaporation of gold onto a template prepared by electron beam lithography (see ref. 37 for detailed fabrication description). The evaporated metal is then attached to a support plate and peeled-off from the template, with the residual electron beam resist etched out using HF. A properly cleaned structure-rinsed in acetone and IPA-had a resonance at $\lambda_{\text {res }}=705 \mathrm{~nm}$. If, however, a thin residue layer formed during the final cleaning, the resonance position could shift by up to $15 \mathrm{~nm}$ to $\lambda_{\text {res }}=720 \mathrm{~nm}$. Due to the nanometer dimensions of the NCs, even a few atomic layers of a dielectric result in an appreciable shift in the resonance wavelength.

For this study we employed a room temperature alumina plasma activated ALD deposition (25 C to avoid deterioration of the NC shape on the gold surface) using an Oxford Instruments FlexAL reactor. The process consisted of the following steps: (1) $30 \mathrm{~ms}$ exposure to trimethylaluminium (TMA) dose at $15 \mathrm{mTorr}$ without any oxygen flow; (2) followed by $1 \mathrm{~s}$ TMA purge and (3) oxygen inflow at $60 \mathrm{sccm}$ for another $1.5 \mathrm{~s}$; (4) with the final $2 \mathrm{~s} 400 \mathrm{~W}$ oxygen plasma oxidation at $15 \mathrm{mTorr}$ with oxygen flow maintained at $60 \mathrm{sccm}$. Throughout the entire process argon gas flow was set at $100 \mathrm{sccm}$.

1. Wood, R. W. On a remarkable case of uneven distribution of light in a diffraction grating spectrum. Proc. Phys. Soc. London 18, 269-275 (1902).

2. Wood, R. W. Optical properties of homogeneous and granular films of sodium and potassium. Phil. Mag. 38, 98-112 (1919).

3. Mills, D. L. \& Weber, M. Enhanced electric fields near gratings: Comments on enhanced raman scattering from surfaces. Phys. Rev. B 26(2), 1075-1078 (1982).

4. Lopez-Rios, T., Mendoza, D., Garcia-Vidal, F. J., Sanchez-Dehesa, J. \& Pannetier, B. Surface shape resonances in lamellar metallic gratings. Phys. Rev. Lett. 81(3), 665-668 (1998)

5. Markel, V. A., Shalaev, V. M., Zhang, P., Huynh, W., Tay, L., Haslett, T. L. \& Moskovits, M. Near-field optical spectroscopy of individual surface-plasmon modes in colloid clusters. Phys. Rev. B 59, 10903-10909 (1999).

6. Le Perchec, J., Quemerais, P., Barbara, A. \& Lopez-Rios, T. Why metallic surfaces with grooves a few nanometers deep and wide may strongly absorb visible light. Phys. Rev. Lett. 100(6), 066408 (2008).

7. Barnes, W. L., Dereux, A. \& Ebbesen, T. W. Surface plasmon subwavelength optics. Nature 424, 824-830 (2003).

8. Garcia de Abajo, F. J. Colloquium: Light scattering by particle and hole arrays. Rev. Mod. Phys. 79(4), 1267-1290 (2007).

9. Willets, K. A. \& Van Duyne, R. P. Localized surface plasmon resonance spectroscopy and sensing. Annu. Rev. of Phys. Chem. 58, 276-297 (2007)

10. Beversluis, M. R., Bouhelier, A. \& Novotny, L. Continuum generation from single gold nanostructures through near-field mediated intraband transitions. Phys. Rev. B 68, 115433 (2003).

11. Castro-Lopez, M., Brinks, D., Sapienza, R. \& van Hulst, N. F. Aluminum for nonlinear plasmonics: Resonance-driven polarized luminescence of al, ag, and au nanoantennas. Nano Lett. 11(11), 4674-4678 (2011).

12. Stockman, M. I., Bergman, D. J., Anceau, C., Brasselet, S. \& Zyss, J. Enhanced second-harmonic generation by metal surfaces with nanoscale roughness: Nanoscale dephasing, depolarization, and correlations. Phys. Rev. Lett. 92(5), 057402 (2004).

13. Fort, E. \& Gresillon, S. Surface enhanced fluorescence. J. Phys. D-Appl. Phys. 41(1), 013001 (2008).

14. Popov, E., Neviere, M., Enoch, S. \& Reinisch, R. Theory of light transmission through subwavelength periodic hole arrays. Phys. Rev. B 62(23), 16100-16108 (2000).

15. Pendry, J. B., Holden, A. J., Robbins, D. J. \& Stewart, W. J. Magnetism from conductors and enhanced nonlinear phenomena. IEEE Trans. Microw. Theory Tech. 47(11), 2075-2084 (1999).

16. Moreau, A., Granet, G., Baida, F. \& Van Labeke, D. Light transmission by subwavelength square coaxial aperture arrays in metallic films. Opt. Express 11, 1131 (2003).

17. Grober, R. D., Schoelkopf, R. J. \& Prober, D. E. Optical antenna: Towards a unity efficiency near-field optical probe. Appl. Phys. Lett. 70, 1354 (1997).

18. Heshmat, B., Li, D., Darcie, T. E. \& Gordon, R. Tuning plasmonic resonances of an annular aperture in metal plate. Optics Express 19(7), 5912-5923 (2011).

19. Bozhevolnyi, S. I., Volkov, V. S., Devaux, E. \& Ebbesen, T. W. Channel plasmonpolariton guiding by subwavelength metal grooves. Phys. Rev. Lett. 95(4), 046802 (2005).

20. Dennis, M. R., Zheludev, N. I. \& Garcia de Abajo, F. J. The plasmon talbot effect. Opt. Express 15(15), 9692-9700 (2007). 
21. Polyakov, A., Cabrini, S., Dhuey, S., Harteneck, B., Schuck, P. J. \& Padmore, H. A. Plasmonic light trapping in nanostructured metal surfaces. Appl. Phys. Lett. 98(20), 203104 (2011).

22. Smith, D. R., Padilla, W. J., Vier, D. C., Nemat-Nasser, S. C. \& Schultz, S. Composite medium with simultaneously negative permeability and permittivity. Phys. Rev. Lett. 84, 4184-4187 (2000).

23. Fan, W., Zhang, S., Minhas, B., Malloy, K. J. \& Brueck, S. R. J. Enhanced infrared transmission through subwavelength coaxial metallic arrays. Phys. Rev. Lett. 94, 033902 (2005).

24. Fromm, D. P., Sundaramurthy, A., Schuck, P. J., Kino, G. \& Moerner, W. E. Gapdependent optical coupling of single bowtie nanoantennas resonant in the visible. Nano Lett. 4(5), 957-961 (2004).

25. Polyakov, A., Thompson, K., Senft, C., Dhuey, S., Harteneck, B., Liang, X., Schuck, J., Cabrini, S., Wan, W. \& Padmore, H. Photocathode performance improvement by plasmonic light trapping in nanostructured metal surfaces. Proc. SPIE - Int. Soc. Opt. Eng. (USA) 8094, 809407 (2011).

26. Zhang, S., Liu, H. \& Mu, G. Electromagnetic enhancement by a single nanogroove in metallic substrate. J. Opt. Soc. Am. A 27(7), 1555-1560 (2010).

27. Raether, H. Surface plasmons on smooth and rough surfaces and on gratings. Springer-Verlag, Berlin, (1988).

28. Atwater, H. A. \& Polman, A. Plasmonics for improved photovoltaic devices. Nat. Mater. 9(3), 205-213 (2010).

29. Homola, J., Yee, S. \& Gauglitz, G. Surface plasmon resonance sensors: review. Sens. Actuator B-Chem. 54(1-2), 3-15 (1999).

30. Polyakov, A., Zolotorev, M., Schuck, P. J. \& Padmore, H. A. Collective behavior of impedance matched plasmonic nanocavities. Opt. Express 20(7), 7685-7693 (2012)

31. Dionne, J. A., Sweatlock, L. A., Atwater, H. A. \& Polman, A. Planar metal plasmon waveguides: frequency-dependent dispersion, propagation, localization, and loss beyond the free electron model. Phys. Rev. B 72(7), 075405 (2005).

32. Leskela, M. \& Ritala, M. Atomic layer deposition (ald): from precursors to thin film structures. Thin Solid Films 409, 138-146 (2002).

33. Groner, M. D., Fabreguette, F. H., Elam, J. W. \& George, S. M. Low-temperature al2o3 atomic layer deposition. Chem. Mater. 16, 639-645 (2004).

34. Profijt, H. B., Potts, S. E., van de Sanden, M. C. M. \& Kessels, W. M. M. Plasmaassisted atomic layer deposition: Basics, opportunities, and challenges. J. Vac. Sci. Technol. A 29(5), 050801 (2011).

35. Ott, A. W., Klaus, J. W., Johnson, J. M. \& George, S. M. Al2o3 thin film growth on si (100) using binary reaction sequencechemistry. Thin Solid Films 292, 135-144 (1997)
36. Kim, S. K., Lee, S. W., Hwang, C. S., Min, Y. S., Won, J. Y. \& Jeong, J. Low temperature $(i$ 100c) deposition of aluminum oxide thin films by ald with 03 as oxidant. J. Electrochem. Soc. 153(5), F69-F76 (2006).

37. Polyakov, A., Padmore, H. A., Liang, X., Dhuey, S., Harteneck, B., Schuck, P. J. \& Cabrini, S. Light trapping in plasmonic nanocavities on metal surfaces. J. Vac. Sci. Technol. B 29(6), 06FF01 (2011).

38. Im, H., Lindquist, N. C., Lesuffleur, A. \& Oh, S.-H. Atomic layer deposition of dielectric overlayers for enhancing the optical properties and chemical stability of plasmonic nanoholes. ACS Nano 4(2), 947-954 (2010)

39. Whitney, A. V., Elam, J. W., Zou, S., Zinovev, A. V., Stair, P. C., Schatz, G. C. \& Van Duyne, R. P. Localized surface plasmon resonance nanosensor: a highresolution distance-dependence study using atomic layer deposition. J. Phys. Chem. B 109, 20522-20528 (2005).

40. Anker, J. N., Hall, W. P., Lyandres, O., Shah, N. C., Zhao, J. \& Van Duyne, R. P. Biosensing with plasmonic nanosensors. Nature Mat. 7, 442-452 (2008).

41. Economou, E. N. Surface plasmons in thin films. Phys. Rev. 182(2), 539-554 (1969).

\section{Acknowledgements}

We thank Roger Falcone for comments, and Dominik Ziegler and Erin Wood for providing the evaporation setup. Portions of this work were performed as a User project at the Molecular Foundry, Lawrence Berkeley National Laboratory, which is supported by the Office of Science, Office of Basic Energy Sciences, of the U.S. Department of Energy under Contract No. DE-AC0205CH11231.

\section{Author contributions}

All authors conceived and designed the research, analyzed the data, worked out the theory, and wrote the manuscript.

\section{Additional information}

Competing financial interests: The authors declare no competing financial interests.

License: This work is licensed under a Creative Commons

Attribution-NonCommercial-NoDerivs 3.0 Unported License. To view a copy of this license, visit http://creativecommons.org/licenses/by-nc-nd/3.0/

How to cite this article: Polyakov, A. et al. Plasmon resonance tuning in metallic nanocavities. Sci. Rep. 2, 933; DOI:10.1038/srep00933 (2012). 